\title{
Percepção de hemiplégicos crônicos sobre o uso de dispositivos auxiliares na marcha
}

\author{
Janaíne Cunha Polese, ${ }^{1}$ Lucas Rodrigues Nascimento, ${ }^{1}$ \\ Christina Danielli Coelho de Morais Faria, ${ }^{1}$ \\ Glória Elizabeth Carneiro Laurentino, ${ }^{2}$ Fátima Rodrigues-de-Paula, ${ }^{1}$ \\ Louise Ada $^{3}$ e Luci Fuscaldi Teixeira-Salmela ${ }^{1}$
}

Como citar Polese JC, Nascimento LR, Faria CDCM, Laurentino GEC, Rodrigues-de-Paula F, Ada L, TeixeiraSalmela LF. Percepção de hemiplégicos crônicos sobre o uso de dispositivos auxiliares na marcha. Rev Panam Salud Publica. 2011:30(3):204-8.

RESUMO Objetivo. Avaliar a percepção de hemiplégicos crônicos sobre o uso de dispositivos auxiliares (DA) na marcha.

Métodos. A partir de um banco de dados de 360 hemiplégicos, foram recrutados 23 indivíduos que utilizavam DA e preenchiam os critérios de inclusão. A média de idade foi de 58,4 anos, tempo pós-acidente vascular encefálico de 80,8 meses e tempo de uso do DA de 67,6 meses. Para avaliar a percepção dos participantes, utilizou-se um questionário padronizado, composto por cinco questões: impacto do DA na habilidade para descarregar peso no membro parético; impacto na habilidade para movimentar o membro parético; impacto na confiança; impacto na segurança; e impacto no jeito de caminhar. As respostas possíveis eram "melhorou", "não alterou" ou "piorou".

Resultados. Quatorze indivíduos utilizavam bengalas e nove utilizavam muletas canadenses; 21 (91,3\%) utilizavam DA somente em vias públicas e dois (8,7\%) utilizavam DA também em ambiente domiciliar. A percepção em relação ao uso do DA foi positiva nas quatro primeiras questões $\left(6,87<\chi^{2}<29,83 ; 0,0001<\mathrm{P} \leq 0,03\right)$, com relatos de melhora na descarga de peso $(82,6 \%)$, na habilidade de movimentar o membro parético $(39,1 \%)$, na confiança para caminhar $(82,6 \%)$ e na segurança $(86,9 \%)$. Não foram observadas diferenças significativas para $o$ item jeito de caminhar $\left(\chi^{2}=1,09 ; \mathrm{P}=0,30\right)$.

Conclusões. A percepção dos indivíduos hemiplégicos crônicos foi positiva em relação ao uso de DA para deambulação, sugerindo que esses dispositivos podem melhorar a mobilidade e a independência durante as atividades de vida diária.

Palavras-chave Acidente cerebral vascular; marcha; bengalas; satisfação do paciente.

A expectativa de vida aumentou nas últimas décadas, especialmente em indivíduos acometidos por doenças crônicas.

1 Universidade Federal de Minas Gerais (UFMG), Departamento de Fisioterapia, Belo Horizonte (MG), Brasil. Correspondência: Luci Fuscaldi Teixeira-Salmela, lfts@ufmg.br

2 Universidade Federal de Pernambuco (UFPE), Departamento de Fisioterapia, Recife (PE), Brasil.

3 The University of Sydney, Faculty of Health Sciences, Sydney, Austrália.
Nesse contexto, o acidente vascular encefálico (AVE) tornou-se um problema de saúde pública (1). O AVE gera deficiências nos domínios de estrutura e função do corpo, podendo causar limitações em atividades e restrições na participação social $(2,3)$.

A restauração da marcha é o objetivo mais frequentemente relatado por indivíduos que sofreram AVE, sendo tam- bém o foco primário dos programas de reabilitação para essa população $(4,5)$. Depois de um AVE, são poucos os indivíduos capazes de se movimentar de modo independente fora de suas casas: aproximadamente $20 \%$ são incapazes de sair de casa sem o uso de algum dispositivo de auxílio à marcha (6). Tendo em vista a grande gama de alterações observadas na marcha pós-AVE, e com o 
intuito de proporcionar um melhor desempenho em atividades de vida diária e garantir maior participação social, alguns profissionais de saúde recomendam o uso de dispositivos auxiliares (DA), como bengalas e muletas canadenses, que conferem maior segurança durante a marcha de indivíduos hemiplégicos $(7,8)$. Esses dispositivos são utilizados por mais de dois terços da população acometida por AVE em algum estágio de recuperação (9), sendo que grande parte dessa população inicia o uso de DA por conta própria.

Entretanto, alguns profissionais da reabilitação ainda são relutantes em relação à prescrição de DA, por acreditar que o seu uso pode limitar a restauração do que eles consideram um "padrão de marcha normal" e a reaquisição de mobilidade independente $(10,11)$. Mais recentemente, diversas publicações mostraram benefícios relacionados ao uso de DA (12-17). A partir disso, um maior número de profissionais passou a prescrever o uso desses dispositivos (18). No entanto, um estudo realizado na Inglaterra mostrou que os profissionais de fisioterapia não costumavam realizar intervenções que encorajassem a independência ou atividade dos pacientes fora do consultório, incluindo a prescrição para uso de DA (19).

Vários estudos investigaram a influência de DA em variáveis biomecânicas da marcha de hemiplégicos (17, 20-22). Entretanto, tais estudos não avaliaram a percepção dos indivíduos em relação ao uso desses dispositivos. É possível, portanto, que, no processo de decisão clínica sobre a prescrição de DA, os profissionais da saúde negligenciem a opinião dos pacientes, o que poderia impactar o processo de adaptação funcional à nova realidade estrutural, ou seja, o uso de um novo dispositivo durante a marcha.

A pesquisa tenta explicar e entender os resultados dos serviços de saúde para proporcionar um melhor atendimento e, assim, um melhor resultado aos usuários (23). Dessa forma, a perspectiva dos indivíduos em relação a determinado desfecho tem um papel essencial no direcionamento das pesquisas (24). Além disso, como afirmam Sumsion e Law (25), a prática baseada em evidências deve estar aliada à prática centrada no cliente, sendo que esta deve reconhecer o papel do cliente na tomada de decisão clínica e ser adequada ao contexto em que os indivíduos vivem. Esse tipo de tomada de decisão pode gerar um aumento da satisfação com os serviços e, consequentemente, maior adesão às recomendações da terapia e melhora dos desfechos funcionais (25). Dessa forma, considerando-se que o desempenho na marcha com o uso do DA poderia ser influenciado pela opinião do paciente acerca da nova realidade estrutural, o objetivo deste estudo foi avaliar a percepção de hemiplégicos crônicos em relação ao uso de DA na marcha.

\section{MATERIAIS E MÉTODOS}

Foi realizado um estudo transversal no Laboratório de Análise de Movimento da Universidade Federal de Minas Gerais (UFMG). Os participantes foram recrutados por telefone a partir de uma lista de 360 hemiplégicos que incluía indivíduos da comunidade em geral e pacientes de ambulatórios de fisioterapia de instituições de ensino de Belo Horizonte, Brasil. Foram selecionados indivíduos que preencheram os seguintes critérios de inclusão: 1) diagnóstico clínico de AVE unilateral, 2) tempo pós-lesão superior a 6 meses, 3) idade igual ou superior a 20 anos, 4) uso habitual de bengalas ou muletas canadenses para caminhar, 5) capacidade de compreender e executar comandos, identificada pelo mini-exame do estado mental com pontos de corte estabelecidos para a população brasileira (26) e 6) ausência de outras deficiências neurológicas ou ortopédicas. O estudo foi aprovado pelo Comitê de Ética em Pesquisa da UFMG e todos os participantes assinaram um termo de consentimento livre e esclarecido concordando com a participação.

Para fins de caracterização da amostra, foram coletados dados demográficos e clínicos referentes a AVE, uso de DA, equilíbrio (por meio da Escala de Equilíbrio de Berg) (27), medo de quedas (28) e estágio de retorno motor (por meio da Escala de Fugl-Meyer — itens para membro inferior) (29).

A percepção dos indivíduos em relação ao uso de DA para deambulação foi avaliada por meio de entrevista direta utilizando um questionário de fácil aplicação desenvolvido por Tyson e Rogerson (13). Engloba cinco questões que avaliam, respectivamente: habilidade para descarregar peso no membro parético; habilidade para movimentar o membro parético durante a deambulação; confiança para caminhar; segurança para caminhar; e jeito de caminhar. As respostas possíveis são: melhorou, não alterou e piorou. $\mathrm{O}$ questionário demonstrou ser válido para avaliar a opinião dos pacientes com relação ao uso de DA (13). Para garantir que todas as questões fossem apresentadas ao paciente da mesma forma, apenas um examinador aplicou o questionário.

\section{Análise dos dados}

Foi utilizada estatística descritiva para a caracterização da amostra quanto às variáveis demográficas e clínicas e para a descrição dos resultados obtidos nas variáveis de desfecho principal do estudo (percepção sobre o DA). Para tanto, foram reportadas medidas de tendência central e de dispersão (média, desvio padrão) para as variáveis quantitativas e medidas de frequência para as variáveis categóricas. Para avaliar se as frequências observadas diferiram do conjunto de frequências esperadas nas questões do questionário sobre o uso do DA, foi utilizado o teste do qui-quadrado $\left(\chi^{2}\right)$ (30). Todas as análises foram realizadas com o Statistical Package for the Social Sciences (SPSS) 15.0, com um nível de significância estabelecido em $<0,05$.

\section{RESULTADOS}

Participaram deste estudo 23 hemiplégicos (13 homens). A média de idade foi de 58,4 anos. Todos os indivíduos iniciaram o uso de DA nos primeiros 6 meses pós-AVE; 14 (60,9\%) indivíduos utilizavam bengalas e nove $(39,1 \%)$ utilizavam muletas canadenses. Observou-se que $21(91,3 \%)$ participantes utilizavam o DA somente em vias públicas, enquanto dois $(8,7 \%)$ utilizavam esse equipamento também em ambiente domiciliar. Em relação à prescrição dos dispositivos auxiliares, 11 participantes $(47,8 \%)$ relataram ter recebido a prescrição de fisioterapeutas; cinco $(21,7 \%)$, de médicos; cinco $(21,7 \%)$ iniciaram o uso por conta própria; e dois $(8,7 \%)$ receberam a prescrição do DA de outro profissional da área da saúde. A síntese dos dados demográficos e clínicos pode ser observada na tabela 1 .

A tabela 2 descreve os resultados relativos à satisfação dos participantes com o DA. O teste do $\chi^{2}$ revelou uma diferença significativa entre as categorias de respostas nas questões "habilidade para descarregar peso no membro parético", "habilidade para movimentar o membro parético", "confiança para caminhar" e 
TABELA 1. Características clínicas e demográficas de 23 hemiplégicos que utilizavam bengala ou muleta canadense para deambular, Belo Horizonte (MG), Brasil, 2010

\begin{tabular}{|c|c|c|c|}
\hline Variável & Média $^{a}$ & $\begin{array}{l}\text { Desvio } \\
\text { padrão }\end{array}$ & $\begin{array}{l}\text { Variação } \\
\text { (mínimo- } \\
\text { máximo) }\end{array}$ \\
\hline Idade (anos) & 58,4 & 8 & $32-70$ \\
\hline Hemiplegia à direita (\%) & $12(52,1)$ & - & - \\
\hline Tempo pós-acidente vascular encefálico (meses) & 80,8 & 43,6 & $24-210$ \\
\hline Tempo de uso do dispositivo auxiliar (meses) & 67,6 & 41,4 & $24-210$ \\
\hline Declararam ter sofrido quedas [No. (\%)] & $14(60,9)$ & - & - \\
\hline \multicolumn{4}{|l|}{ Medo de cair [No. (\%)] } \\
\hline Muito medo & $10(43,5)$ & - & - \\
\hline Medo moderado & $3(13,1)$ & - & - \\
\hline Pouco medo & $1(4,3)$ & - & - \\
\hline Nenhum medo & $9(39,1)$ & - & - \\
\hline Escore de recuperação motorab & 23 & 5,5 & $15-31$ \\
\hline Escore de equilíbrioc & 48,4 & 4,5 & $39-56$ \\
\hline
\end{tabular}

"segurança para caminhar". A primeira opção de resposta (melhorou) foi a que mais contribuiu para a significância encontrada $\left(6,87<\chi^{2}<29,83 ; 0,0001<P \leq\right.$ $0,03)$. Não foram observadas diferenças estatisticamente significativas nas respostas relacionadas ao jeito de caminhar $\left(\chi^{2}=1,09 ; P=0,30\right)$. Observou-se que nenhum dos participantes avaliados relatou piora em relação à habilidade para descarregar peso no membro parético, à confiança para caminhar e ao jeito de caminhar. Do total da amostra estudada, apenas dois indivíduos relataram piora na habilidade para movimentar o membro parético e apenas um indivíduo relatou piora na segurança para caminhar.

\section{DISCUSSÃO}

Não foram encontrados estudos prévios que tenham investigado a opinião de indivíduos hemiplégicos crônicos brasileiros acerca da influência do uso de bengalas e muletas canadenses durante a marcha. No Reino Unido, Tyson e Rogerson (13) avaliaram o efeito imediato do uso de DA na mobilidade funcional, assim como a opinião de hemiplégicos agudos pós-AVE acerca desse efeito. Como no presente estudo, os resultados demonstraram que os indivíduos responderam positivamente ao uso do DA, relatando maior confiança e segurança, além de melhora na marcha.

Neste estudo, todos os indivíduos iniciaram a utilização dos DA nos primeiros 6 meses pós-lesão e, mesmo realizando programas de reabilitação, não adquiriram a marcha comunitária (fora do ambiente domiciliar) sem o uso dos dispositivos. Nesse sentido, estudos relataram que o uso de DA pode melhorar a mobilidade de indivíduos hemiplégicos quando esses dispositivos são prescritos durante a reabilitação $(16,20,31,32)$, como adjuvantes ao tratamento fisioterápico. Aproximadamente metade dos indivíduos $(47,8 \%)$ em nosso estudo recebeu a prescrição do DA por um fisioterapeuta; é possível que os profissionais de uma forma geral não acreditassem haver indicação para uso do DA, ou ainda que alguns profissionais relutassem em prescrever esses equipamentos. Já Hamzat e Koribi (8) observaram que $76 \%$ dos hemiplégicos avaliados receberam a prescrição do DA por fisioterapeutas, e que o restante da amostra adquiriu o DA por conta própria, por entender que o uso dos mesmos poderia prevenir quedas.

Observou-se, na aplicação da escala relacionada à opinião dos hemiplégicos, que a opção de resposta "melhorou" foi a que mais contribuiu para a significância encontrada, exceto na questão cinco. Dessa forma, pode-se inferir que a amostra estudada teve uma visão positiva em relação ao uso de bengalas ou muletas canadenses durante a marcha.

Em relação à primeira questão do questionário, pôde-se observar que a maioria dos indivíduos relatou maior percepção de descarga de peso no membro parético com o uso do DA. A literatura é controversa acerca desse tópico. Achados prévios indicaram não haver diferenças na descarga de peso entre os membros com o uso de bengalas ou muletas (33), enquanto outros demonstraram melhora da simetria entre a descarga de peso entre os membros inferiores (34). Entretanto, estudos mais recentes ressaltaram que a simetria em hemiplégicos não está relacionada a um melhor desempenho em atividades funcionais (35-38). Na verdade, não há evidência de que a simetria desempenhe algum papel em promover desempenho funcional e, de fato, variáveis assimétricas parecem ser mais relevantes para desfechos funcionais, tais como variáveis espaço-temporais e velocidade (38).

Neste estudo, observou-se que apenas um indivíduo relatou piora da confiança e da segurança durante a deambulação com bengalas e muletas, sendo que a maioria $(82,6 \%$ e $86,9 \%$, respectivamente)

TABELA 2. Satisfação com o uso de dispositivos auxiliares em 23 hemiplégicos que utilizavam bengala ou muleta canadense para deambular, Belo Horizonte (MG), Brasil, 2010a

\begin{tabular}{|c|c|c|c|c|}
\hline \multirow[b]{2}{*}{ Item } & \multicolumn{3}{|c|}{ No. (\%) } & \multirow[b]{2}{*}{$P$ resíduos ${ }^{b}$} \\
\hline & Melhorou & Não alterou & Piorou & \\
\hline Habilidade para descarregar peso no membro parético & $19(82,6)$ & $4(17,4)$ & $0(0)$ & $0,002 / 7,5$ \\
\hline Habilidade para movimentar membro parético na deambulação & $9(39,1)$ & $12(52,2)$ & $2(8,7)$ & $0,03 / 4,3$ \\
\hline Confiança para caminhar & $19(82,6)$ & $4(17,4)$ & $0(0)$ & $0,002 / 7,5$ \\
\hline Segurança para caminhar & $20(86,9)$ & $2(8,7)$ & $1(4,3)$ & $0,0001 / 12,3$ \\
\hline Jeito de caminhar & $14(60,9)$ & $9(39,1)$ & $0(0)$ & $0,29 / 2,5$ \\
\hline
\end{tabular}

a Os indivíduos responderam à seguinte questão: "O uso da bengala/muleta mudou o seu/sua. ...."

b Resíduo: diferença entre os valores observados e esperados (espera-se que os valores sejam iguais dentre as opções de resposta, ou seja, 33,3\%). 
relatou sentir melhora nesses dois itens. Contrariamente, Hamzat e Kobiri (8) observaram, em 50 hemiplégicos agudos e crônicos, que aqueles que faziam uso de bengalas apresentavam menor participação social e pior equilíbrio do que aqueles que não as utilizavam. Esses achados poderiam ser explicados pelo fato de que os indivíduos na fase aguda do AVE não tinham a função da marcha estabelecida, o que pode ter interferido na menor participação social, já que a marcha é um pré-requisito para diversas atividades de vida diária (39).

Apenas dois indivíduos relataram piora na questão relacionada à habilidade para movimentar o membro parético, e apenas um relatou piora na questão relacionada à segurança para caminhar: esses participantes relataram quedas nos últimos 6 meses, além de sentir muito medo de cair. No entanto, esses indivíduos apresentaram um escore acima do ponto de corte na Escala de Equilíbrio de Berg (46 pontos), e ainda ressaltaram não pretender deixar de usar a bengala, reforçando a hipótese de que o uso de DA pode ser um facilitador para a marcha, acarretando maior participação social.
Nenhum indivíduo relatou piora do jeito de caminhar com o uso do DA. Assim, refutando a ideia de alguns terapeutas $(10,11)$, os resultados deste estudo mostraram que os indivíduos hemiplégicos crônicos não têm como prioridade a restauração da marcha com "padrão estético normal" sem o uso de bengalas ou muletas; sua prioridade parece ser restabelecer a função da marcha, mesmo que com a estética adaptada (18), reforçando a importância do uso da prática centrada no cliente para a tomada de decisão clínica (25).

$\mathrm{O}$ pequeno número da amostra justifica-se pelo fato de poucos hemiplégicos crônicos advindos do banco original (composto por cerca de 360 indivíduos pós-AVE) terem recebido prescrição de bengalas e muletas canadenses e continuarem em uso das mesmas no momento do estudo. Dessa forma, todos aqueles que corresponderam aos critérios de inclusão do presente estudo foram recrutados, totalizando 23 indivíduos. Os resultados, no entanto, impedem conclusões definitivas, devido ao delineamento do estudo e à composição da amostra, que incluiu indivíduos adaptados ao uso do
DA, no estágio crônico e com altos níveis funcionais.

Em resumo, os indivíduos hemiplégicos crônicos que compuseram a amostra do presente estudo manifestaram uma percepção positiva em relação ao uso de DA para deambulação, sugerindo que o uso desses dispositivos pode auxiliar na mobilidade e independência durante atividades de vida diária, podendo acarretar uma maior participação social. Considerando a amostra do presente estudo, constituída por participantes que relataram a necessidade de uso do DA principalmente em vias públicas, pode-se inferir que a percepção de melhora é modulada por aspectos relacionados a fatores sociais e estrutura física do ambiente, sendo o DA um facilitador do desempenho na marcha.

Agradecimentos. O estudo recebeu apoio do Conselho Nacional de Desenvolvimento Científico e Tecnológico (CNPq), Coordenação de Aperfeiçoamento de Pessoal de Nível Superior (CAPES) e Fundação de Amparo à Pesquisa do Estado de Minas Gerais (FAPEMIG).

\section{REFERÊNCIAS}

1. Saposnik G, Del Brutto OH, Iberoamerican Society of Cerebrovascular Diseases. Stroke in South America: A systematic review of incidence, prevalence, and stroke subtypes. Stroke. 2003;34(9):2103-7.

2. Salter K, Jutai JW, Teasell R, Foley NC, Bitensky J, Bayley M. Issues for selection of outcome measures in stroke rehabilitation: ICF activity. Disabil Rehabil. 2005;27(6):315-40.

3. Salter K, Jutai JW, Teasell R, Foley NC, Bitensky J, Bayley M. Issues for selection of outcome measures in stroke rehabilitation: ICF Participation. Disabil Rehabil. 2005;27(9): 507-28.

4. Jorgensen HS, Nakayama H, Raaschou HO, Olsen TS. Recovery of walking function in stroke patients: the Copenhagen Stroke Study. Arch Phys Med Rehabil. 1995;76(1):27-32.

5. Bohannon RW, Andrews AW, Smith MB. Rehabilitation goals of patients with hemiplegia. Int J Rehabil Res. 1988;11(2):181-3.

6. Lord SE, McPherson K, McNaughton HK, Rochester L, Weatherall M. Community ambulation after stroke: how important and obtainable is it and what measures appear predictive? Arch Phys Med Rehabil. 2004;85(2): 234-9.

7. Laufer Y. Effects of one-point and four-point canes on balance and weight distribution in patients with hemiparesis. Clin Rehabil. 2002; 16(2):141-8.
8. Hamzat TK, Kobiri A. Effects of walking with a cane on balance and social participation among community-dwelling post-stroke individuals. Eur J Phys Rehabil Med. 2008;44(2):121-6.

9. Organização Mundial da Saude. Classificação Internacional de Incapacidade, Funcionalidade e Saúde - CIF. São Paulo: EDUSP; 2003.

10. Davidson I. Physiotherapists working with stroke patients: a national survey. Physiother. 2000;86(2):69-80.

11. Lennon S, Baxter D, Ashburn A. Physiotherapy based on the Bobath concept in stroke rehabilitation: a survey within the UK. Disabil Rehabil. 2001;23(6):254-62.

12. Gosman-Hedstrom G, Claesson L, Blomstrand C. Assistive devices in elderly people after stroke: a longitudinal, randomized study The Göteborg $70+$ stroke study. Scand J Occup Ther. 2002;9(3):109-18.

13. Tyson SF, Rogerson L. Assistive walking devices in non-ambulant patients undergoing rehabilitation after stroke: the effects on functional mobility, walking impairments, and patients' opinion. Arch Phys Med Rehabil. 2009; 90(3):475-9.

14. Agree EM. The influence of personal care and assistive devices on the measurement of disability. Soc Sci Med. 1999;48(4):427-43.

15. Stowe S, Hopesb J, Mulley G. Gerotechnology series: 2. Walking aids. Eur Geriatr Med. 2010; 1(2):122-7.
16. Kuan TS, Tsou JY, Su FC. Hemiplegic gait of stroke patients: the effect of using a cane. Arch Phys Med Rehabil. 1999;80(7):777-84.

17. Chen CL, Chen HC, Wong MK, Tang FT, Chen RS. Temporal stride and force analysis of cane-assisted gait in people with hemiplegic stroke. Arch Phys Med Rehabil. 2001; 82(1):43-8.

18. Lennon S, Ashburn A. The Bobath concept in stroke rehabilitation: a focus group study of the experienced physiotherapists' perspective. Disabil Rehabil. 2000;22(15):665-74.

19. Tyson S, Selley A. A content analysis of physiotherapy for postural control in people with stroke: an observational study. Disabil Rehabil. 2006;28(13-14):865-72.

20. Tyson SF. The influence of walking aids on hemiplegic gait. Physiother Theory Pract. 1994;10:77-86.

21. Tyson SF. Trunk kinematics in hemiplegic gait and the effect of walking aids. Clin Rehabil. 1999;13(4):295-300.

22. Lai MS. The effect of walking aids on gait symettry and speed in hemiplegic patients [dissertação]. Chester: University of Chester; 2008.

23. Gonçalves Campolina A, Mesquita Ciconelli R. Qualidade de vida e medidas de utilidade: parâmetros clínicos para as tomadas de decisão em saúde. Rev Panam Salud Publica. 2006;19(2):128-36. 
24. Cieza A, Geyh S, Chatterji S, Kostanjsek N, Ustun B, Stucki G. ICF linking rules: an update based on lessons learned. J Rehabil Med. 2005;37(4):212-8.

25. Sumsion T, Law M. A review of evidence on the conceptual elements informing clientcentred practice. Can J Occup Ther. 2006; 73(3):153-62.

26. Bertolucci PH, Brucki SM, Campacci SR, Juliano Y. O mini-exame do estado mental em uma população geral: impacto da escolaridade. Arq Neuropsiquiatr. 1994;52(1):1-7.

27. Miyamoto ST, Lombardi Junior I, Berg KO, Ramos LR, Natour J. Brazilian version of the Berg balance scale. Braz J Med Biol Res. 2004; 37(9):1411-21.

28. Zecevic AA, Salmoni AW, Speechley M, Vandervoort AA. Defining a fall and reasons for falling: comparisons among the views of seniors, health care providers, and the research literature. Gerontologist. 2006; 46(3):367-76.

29. Maki T, Quagliato EM, Cacho EW, Paz LP, Nascimento NH, Inoue MM, et al. Estudo da confiabilidade da aplicação da escala de FuglMeyer no Brasil. Rev Bras Fisioter. 2006;10(2): 177-83.
30. Portney LG, Watkins MP. Foundations of clinical research: Appications to practice. 3a. ed. Upper Saddle River: Pearson PrenticeHall; 2009.

31. Hesse S, Jahnke MT, Schaffrin A, Lucke D, Reiter F, Konrad M. Immediate effects of therapeutic facilitation on the gait of hemiparetic patients as compared with walking with and without a cane. Electroencephalogr Clin Neurophysiol. 1998;109(6):515-22.

32. Buurke JH, Hermens HJ, Erren-Wolters CV, Nene AV. The effect of walking aids on muscle activation patterns during walking in stroke patients. Gait Posture. 2005;22(2):164-70.

33. Laufer $Y$. The effect of walking aids on balance and weight-bearing patterns of patients with hemiparesis in various stance positions. Phys Ther. 2003;83(2):112-22.

34. Beauchamp MK, Skrela M, Southmayd D, Trick J, Kessel MV, Brunton K, et al. Immediate effects of cane use on gait symmetry in individuals with subacute stroke. Physiother Can. 2009;61(3):154-60.

35. Faria CD, Teixeira-Salmela LF, Nadeau S. Effects of the direction of turning on the timed up \& go test with stroke subjects. Top Stroke Rehabil. 2009;16(3):196-206.
36. Teixeira-Salmela LF, Lima RC, Lima LA, Morais SG, Goulart F. Assimetria e desempenho funcional em hemiplégicos crônicos antes e após programa de treinamento em academia. Rev Bras Fisioter. 2005;9(2):227-33.

37. Faria CD, Reis DA, Teixeira-Salmela LF, Nadeau S. Desempenho de hemiplégicos no giro de $180^{\circ}$ realizado em direção ao lado parético e não parético antes e após um programa de treinamento. Rev Bras Fisioter. 2009; 13(5):451-7.

38. Griffin MP, Olney SJ, McBride ID. Role of symmetry in gait performance of stroke subjects with hemiplegia. Gait Posture. 1995;3(3):132-42.

39. Prajapati SK, Gage WH, Brooks D, Black SE, Mcllroy WE. A novel approach to ambulatory monitoring: Investigation into the quantity and control of everyday walking in patients with subacute stroke. Neurorehabil Neural Repair. 2011;25(1):6-14. em versão revisada em 20 de março de 2011.

ABSTRACT Objective. To assess the perception of patients with chronic hemiplegia regarding the use of assistive walking devices (AWD).

\section{Perception of patients with chronic hemiplegia regarding the use of assistive walking devices}

Methods. Twenty-three individuals who met the inclusion criteria and used AWD were recruited from a database of 360 stroke survivors. Their mean age was 58.4 years, mean time since stroke was 80.8 months, and mean time using AWD was 67.6 months. To assess the participants' perception, was used a standardized questionnaire covering the impact of AWDs on five aspects: weight-bearing on the paretic limb; ability to move the paretic limb; confidence; safety; and walking style. Possible answers were "improved," "unchanged," or "decreased."

Results. Fourteen individuals used canes and nine used elbow crutches; 21 (91.3\%) used AWDs on public roads and only two (8.7\%) used AWDs at home. The perception regarding the use of AWDs were positive in the first four questions $\left(6.87<\chi^{2}\right.$ $<29.83 ; 0.0001<P \leq 0.03)$, with reports of improvement in weight-bearing $(82.6 \%)$, ability to move the paretic limb $(39.1 \%)$, confidence $(82.6 \%)$, and safety $(86.9 \%)$. No significant differences were found in terms of walking style $\left(\chi^{2}=1.09 ; P=0.30\right)$.

Conclusions. The perception of chronic hemiplegic subjects regarding the use of AWD were positive, suggesting that these devices can improve mobility and independence in activities of daily living.

Key words Stroke; gait; cane; patient satisfaction. 